\title{
Finite groups are big as semigroups
}

\author{
I. Dolinka and N. Ruškuc
}

Dedicated to the memory of Jaroslav Ježek (1945-2011)

\begin{abstract}
We prove that a finite group $G$ occurs as a maximal proper subsemigroup of an infinite semigroup (in the terminology of Freese, Ježek and Nation, $G$ is a big semigroup) if and only if $|G| \geq 3$. In fact, any finite semigroup whose minimal ideal contains a subgroup with at least three elements is big.
\end{abstract}

Mathematics Subject Classification (2010). Primary 20M10; Secondary 20F05, 20F50.

Keywords. Finite maximal subsemigroup, Rees matrix semigroup.

\section{Introduction}

Let $\mathcal{K}$ be a class of algebraic structures of a given similarity type. Following the extensive study [3], we say that a finite structure $B \in \mathcal{K}$ is $\mathcal{K}$-big if there exists a countably infinite structure $A \in \mathcal{K}$ such that $B$ is a maximal proper substructure of $A$. Equivalently, $A$ is (finitely) generated by $B$ and any single element from $A \backslash B$. In [3], Freese, Ježek and Nation studied and characterised all big lattices: they produced a complete list consisting of 145 minimal big lattices (there are 81 of them up to a dual isomorphism) and proved that a finite lattice is big if and only if it contains one of these as a sublattice.

For groups, the (open) question of which finite groups are big is closely connected to Burnside-type problems. In [6], Ol'shanskil constructed, for any prime $p>10^{75}$, a 2-generated infinite group $G$ such that each proper subgroup of $G$ has order $p$, thus exhibiting the first explicit example of a Tarski monster group, and showing, in particular, that any cyclic group $C_{p}$ is big for a sufficiently large prime $p$. Later, this was improved by Adyan and Lysionok [1]: for any odd integer $n \geq 1003$ they constructed an infinite 2-generated

The research of the first author is supported by the Ministry of Education and Science of the Republic of Serbia through Grant No.174019, and by a grant (Contract 114-4512002/2011) of the the Secretariat of Science and Technological Development of the Autonomous Province of Vojvodina. 
group $G$ with the property that any proper subgroup of $G$ is contained in a cyclic subgroup of order $n$. Therefore, any cyclic group $C_{2 k+1}$ with $k \geq 501$ is a big group; in fact, this group is even $\mathcal{B}_{2 k+1}$-big, where $\mathcal{B}_{2 k+1}$ is the Burnside variety of all groups of exponent $2 k+1$.

In this paper we initiate the study of big semigroups and their properties. In particular, we obtain the following full description of finite groups that occur as maximal subsemigroups of countably infinite semigroups.

Theorem A. Let $G$ be a finite group. Then $G$ is big with respect to the class of all semigroups if and only if $|G| \geq 3$.

In the next section, we gather the necessary semigroup-theoretical preliminaries and formulate our main result, Theorem B below, which is slightly more general than the above statement; the backward implication in Theorem A will follow as a corollary. In Sec. 3 we describe the construction utilised in our proofs, which are presented in Sec. 4 and 5. Along the way, we provide a few valuable pieces of information on the structure of infinite semigroups entertaining finite semigroups as maximal subsemigroups.

\section{Preliminaries}

We refer to $[2,5]$ for a general semigroup-theoretical background; however, we recall several key notions in order to keep the note mostly self-contained.

An ideal of a semigroup $S$ is a non-empty subset $I \subseteq S$ such that $t s, s t \in I$ for any $t \in I$ and $s \in S$. For an arbitrary element $a \in S$ of a semigroup $S$ it is clear that the (principal) ideal generated by $a$, the least ideal of $S$ containing $a$, is $S^{1} a S^{1}=\left\{s a t: s, t \in S^{1}\right\}$, where $S^{1}$ denotes the monoid obtained by extending $S$ by a new symbol 1 acting as an identity element. In products, such as in sat, the condition $s, t \in S^{1}$ indicates the possibility that some of the factors $s, t$ may not be present (so, $S^{1} a S^{1}$ is in fact a short-hand for $\{a\} \cup a S \cup S a \cup S a S$ ). Naturally, the elements of $S$ can be classified according to the principal ideal they generate, thus giving rise to Green's relation $\mathscr{J}$ defined for $a, b \in S$ by

$$
a \mathscr{J} b \quad \text { if and only if } S^{1} a S^{1}=S^{1} b S^{1} .
$$

The $\mathscr{J}$-class of $a$ is denoted by $J_{a}$. Furthermore, a partial order can be defined on the set of $\mathscr{J}$-classes by $J_{a} \leq J_{b}$ if and only if $S^{1} a S^{1} \subseteq S^{1} b S^{1}$; obviously, this does not depend on the choice of representatives $a, b$. The latter condition is also equivalent to the existence of $s, t \in S^{1}$ such that $a=s b t$; so, the $\mathscr{J}$-order is actually the divisibility relation in a semigroup. Hence, any subgroup $G$ of a semigroup $S$ is entirely contained in a suitable $\mathscr{J}$-class, since all elements of $G$ are divisible by each other.

A semigroup is simple if it has no proper ideals. Clearly, a simple semigroup must consist of a single $\mathscr{J}$-class. Conversely, any minimal (with respect to the order introduced above) $\mathscr{J}$-class of $S$ is a simple subsemigroup of $S$; in fact, this is the single minimal ideal of $S$ (the kernel of $S$ ), showing that 
there can be at most one minimal $\mathscr{J}$-class in a semigroup. Note that any finite semigroup has a kernel.

If $S$ is a finite semigroup that is (isomorphic to) a maximal proper subsemigroup of a countably infinite semigroup $T$, then $T$ will be called a witness for $S$, as it certifies $S$ as a big semigroup.

Lemma 1. Assume that $S$ is a big semigroup, and let $T$ be any witness for $S$. Then $T \backslash S$ is contained in a single $\mathscr{J}$-class of $T$. In particular, if $S$ is a group, then $T$ has at most two $\mathscr{J}$-classes.

Proof. Let $a, b \in T \backslash S$. Since the set $S \cup\{a\}$ must generate $T$, there exists a word $\mathbf{w}=\mathbf{w}\left(x_{1}, x_{2}, \ldots, x_{n+1}\right)$ such that

$$
b=\mathbf{w}\left(a, s_{1}, \ldots, s_{n}\right)
$$

holds in $T$ for suitable $s_{1}, \ldots, s_{n} \in S$. Here $x_{1}$ makes at least one occurrence in $\mathbf{w}$ (i.e. it is not a dummy letter), for otherwise $b \in S$, which is not the case. Therefore, $a$ appears as a factor in the product $\mathbf{w}\left(a, s_{1}, \ldots, s_{n}\right)$, and so $J_{a} \leq J_{b}$. By reversing the roles of $a$ and $b$, we conclude that $J_{b} \leq J_{a}$, thus $a \mathscr{J} b$.

An analogous fact holds for maximal subsemigroups of finite semigroups as well, see the note of Graham et al. [4].

The notions of an ideal and the associated equivalence $\mathscr{J}$ can be made one-sided. Namely, the subset $I \subseteq S$ of a semigroup $S$ is called a left (right) ideal of $S$ if $s t \in I(t s \in I)$ for all $t \in I, s \in S$. The left (right) ideal generated by $a \in S$ is $S^{1} a\left(a S^{1}\right)$, so one can define the following equivalences on $S$ :

$$
\begin{array}{ll}
a \mathscr{L} b & \text { if and only if } \\
a \mathscr{R} b & S^{1} a=S^{1} b, \\
\text { if and only if } & a S^{1}=b S^{1} .
\end{array}
$$

The order of left (right) principal ideals by inclusion induces an order on the set of all $\mathscr{L}$ - $\left(\mathscr{R}\right.$-) classes $L_{a}\left(R_{a}\right)$ of $S$. Clearly, a minimal $\mathscr{L}$ - $(\mathscr{R}$-)class in this order (which need not to be unique) is a minimal left (right) ideal of $S$.

Our main result is now as follows.

Theorem B. Let $S$ be a finite semigroup. If the kernel of $S$ contains a subgroup $G$ such that $|G| \geq 3$, then $S$ is big.

In particular, since any group $G$ is easily seen to be simple (so $G$ coincides with its kernel), the above theorem implies that any finite group $G$ with $|G| \geq 3$ is big as a semigroup.

The observation contained in Lemma 1 will serve as an initial lead in our construction. Namely, given a finite semigroup $S$ satisfying the conditions of Theorem B, we are going to construct a semigroup $\Sigma_{S}$ containing $S$, so that $S$ acts on the simple ideal of $\Sigma_{S}$. The latter ideal will be constructed from a 2-generated infinite periodic group (that is, a counterexample to the Burnside conjecture). 


\section{The construction}

One of the fundamental concepts in semigroup theory is that of a Rees matrix semigroup $\mathcal{M}(I, G, \Lambda, P)$. Its parameters are: a group $G$ (called the structure group), two index sets $I, \Lambda$, and the sandwich matrix $P=\left[p_{\mu, i}\right]_{\Lambda \times I}$, whose entries are from $G$. Its elements are triples of the form $(i, g, \mu)$, where $i \in I$, $g \in G$ and $\mu \in \Lambda$; the multiplication is defined by

$$
(i, g, \mu)(j, h, \nu)=\left(i, g p_{\mu, j} h, \nu\right) .
$$

Every Rees matrix semigroup is completely simple, which means that it is simple and contains a primitive idempotent (an idempotent that is minimal in the so-called Rees order on the set $E(S)$ of all idempotents of a semigroup $S$ defined by $e \leq f$ is and only if $e=e f=f e)$. Conversely, by the ReesSuškevič Theorem $[2,5]$, every completely simple (and, in particular, every finite simple) semigroup is isomorphic to a Rees matrix semigroup.

It follows that the kernel of a finite semigroup must be of this form as well; a minimal left (right) ideal of such a kernel is a set of the form $I \times G \times\{\mu\}$ for some $\mu \in \Lambda$ (resp. $\{i\} \times G \times \Lambda$ for some $i \in I$ ), while the maximal subgroups are formed by sets of the form $G_{i, \mu}=\{i\} \times G \times\{\mu\}$, $i \in I, \mu \in \Lambda$. Thus the conditions of Theorem B postulate that in the Rees matrix representation of the kernel of $S$ we have $|G| \geq 3$. So, let us fix one such maximal subgroup: let $e \in E(S)$ be its identity element, and let $L$ and $R$ be the (unique) minimal left and right ideals of $S$, respectively, containing $G$. One instantly has $L=S e=L_{e}$ and $R=e S=R_{e}$; denote by $G_{e}=L \cap R$ the considered maximal subgroup of the kernel of $S$. Also, we fix distinct $c, d \in G_{e} \backslash\{e\}$.

In our construction we will have

$$
\Sigma_{S}=S \cup \mathcal{M}(L, H, R, P),
$$

with $\mathcal{M}(L, H, R, P)$ being the kernel of $\Sigma_{S}$. For the structure group $H$ of the newly constructed $\mathscr{J}$-class we take an arbitrary but fixed infinite periodic 2-generated group, $H=\left\langle\gamma_{1}, \gamma_{2}\right\rangle$.

To specify the sandwich matrix $P=\left[p_{a, b}\right]_{R \times L}$ and the multiplication of elements of $S$ by the elements of $M$, we fix a mapping $\lambda: L \cup R \rightarrow H$ with the following properties:

(1) $\lambda(e)=1_{H}$;

(2) $\lambda(c)=\gamma_{1}$;

(3) $\lambda(d)=\gamma_{2}$;

(4) $\lambda($ se $)=\lambda($ ese $)$ for any $s \in S$.

Such a mapping exists because the conditions (1)-(3) define the values of $\lambda$ on three specific elements of $R$, while the condition (4) simply postulates that the values of $\lambda$ on $L=S e$ are uniquely determined by the values that $\lambda$ takes on $G_{e}=e S e$.

Now define, for any $a \in R, b \in L$ :

$$
p_{a, b}=\lambda(a)^{-1} \lambda(a b) \lambda(b)^{-1} .
$$


For any $\ell \in L$ we have $\ell e=\ell$, and hence

$$
p_{e, \ell}=\lambda(e)^{-1} \lambda(e \ell) \lambda(\ell)^{-1}=\lambda(e \ell e) \lambda(\ell e)^{-1}=1_{H},
$$

using (1), (4). The multiplication between elements of $S$ and $\mathcal{M}(L, H, R, P)$ is defined by

$$
\begin{aligned}
& s(\ell, h, r)=\left(s \ell, \lambda(s \ell) \lambda(\ell)^{-1} h, r\right), \\
& (\ell, h, r) s=\left(\ell, h \lambda(r)^{-1} \lambda(r s), r s\right),
\end{aligned}
$$

for all $s \in S, \ell \in L, r \in R$ and $h \in H$.

\section{Proof of Theorem B}

Lemma 2. The groupoid $\Sigma_{S}$, defined in the previous section, is a semigroup.

Proof. Since both $S$ and $\mathcal{M}(L, H, R, P)$ are semigroups, the associativity need only be checked in the case of a product in which two factors are from one 'component' of $\Sigma_{S}$ (that is, $S$ or $\mathcal{M}(L, H, R, P)$ ), and one is from the other. In the following, let $a, b \in S, \ell, \ell_{1}, \ell_{2} \in L, r, r_{1}, r_{2} \in R$ and $h, h^{\prime} \in H$.

First of all,

$$
(a(\ell, h, r)) b=\left(a \ell, \lambda(a \ell) \lambda(\ell)^{-1} h \lambda(r)^{-1} \lambda(r b), r b\right)=a((\ell, h, r) b) .
$$

Next,

$$
\begin{aligned}
a(b(\ell, h, r)) & =a\left(b \ell, \lambda(b \ell) \lambda(\ell)^{-1} h, r\right)=\left(a b \ell, \lambda(a b \ell) \lambda(b \ell)^{-1} \lambda(b \ell) \lambda(\ell)^{-1} h, r\right) \\
& =\left(a b \ell, \lambda(a b \ell) \lambda(\ell)^{-1} h, r\right)=(a b)(\ell, h, r)
\end{aligned}
$$

and, similarly, $((\ell, h, r) a) b=(\ell, h, r)(a b)$. Also, we have

$$
\begin{aligned}
\left(a\left(\ell_{1}, h, r_{1}\right)\right)\left(\ell_{2}, h^{\prime}, r_{2}\right) & =\left(a \ell_{1}, \lambda\left(a \ell_{1}\right) \lambda\left(\ell_{1}\right)^{-1} h p_{r_{1}, \ell_{2}} h^{\prime}, r_{2}\right) \\
& =a\left(\left(\ell_{1}, h, r_{1}\right)\left(\ell_{2}, h^{\prime}, r_{2}\right)\right)
\end{aligned}
$$

and, similarly, $\left(\left(\ell_{1}, h, r_{1}\right)\left(\ell_{2}, h^{\prime}, r_{2}\right)\right) a=\left(\ell_{1}, h, r_{1}\right)\left(\left(\ell_{2}, h^{\prime}, r_{2}\right) a\right)$. Finally,

$$
\begin{aligned}
\left(\left(\ell_{1}, h, r_{1}\right) a\right)\left(\ell_{2}, h^{\prime}, r_{2}\right) & =\left(\ell_{1}, h \lambda\left(r_{1}\right)^{-1} \lambda\left(r_{1} a\right), r_{1} a\right)\left(\ell_{2}, h^{\prime}, r_{2}\right) \\
& =\left(\ell_{1}, h \lambda\left(r_{1}\right)^{-1} \lambda\left(r_{1} a\right) p_{r_{1} a, \ell_{2}} h^{\prime}, r_{2}\right) \\
& =\left(\ell_{1}, h \lambda\left(r_{1}\right)^{-1} \lambda\left(r_{1} a\right) \lambda\left(r_{1} a\right)^{-1} \lambda\left(r_{1} a \ell_{2}\right) \lambda\left(\ell_{2}\right)^{-1} h^{\prime}, r_{2}\right) \\
& =\left(\ell_{1}, h \lambda\left(r_{1}\right)^{-1} \lambda\left(r_{1} a \ell_{2}\right) \lambda\left(\ell_{2}\right)^{-1} h^{\prime}, r_{2}\right) \\
& =\left(\ell_{1}, h \lambda\left(r_{1}\right)^{-1} \lambda\left(r_{1} a \ell_{2}\right) \lambda\left(a \ell_{2}\right)^{-1} \lambda\left(a \ell_{2}\right) \lambda\left(\ell_{2}\right)^{-1} h^{\prime}, r_{2}\right) \\
& =\left(\ell_{1}, h p_{r_{1}, a \ell_{2}} \lambda\left(a \ell_{2}\right) \lambda\left(\ell_{2}\right)^{-1} h^{\prime}, r_{2}\right) \\
& =\left(\ell_{1}, h, r_{1}\right)\left(a \ell_{2}, \lambda\left(a \ell_{2}\right) \lambda\left(\ell_{2}\right)^{-1} h^{\prime}, r_{2}\right) \\
& =\left(\ell_{1}, h, r_{1}\right)\left(a\left(\ell_{2}, h^{\prime}, r_{2}\right)\right)
\end{aligned}
$$

where we have repeatedly used the form (3.1) of the entries of $P$ and the multiplication rules (3.2) and (3.3). Hence, the lemma is proved. 
As an alternative approach, the fact that $\Sigma_{S}$ is a semigroup can be derived from the general results of [7] on ideal extensions of Rees matrix semigroups.

Proof of Theorem B. Let $\ell \in L, r \in R$ and $h_{0} \in H$ be arbitrary; we want to prove that the subsemigroup $T$ generated by $S \cup\left\{\left(\ell, h_{0}, r\right)\right\}$ is actually equal to the whole $\Sigma_{S}$. Notice that there is no loss of generality in assuming that $\ell=r=e$, for otherwise the fact that $L$ and $R$ are minimal left and right ideals of $S$, respectively, implies that $e=s \ell=r t$ for some $s, t \in S$, thus

$$
s\left(\ell, h_{0}, r\right) t=\left(e, \lambda(s \ell) \lambda(\ell)^{-1} h_{0} \lambda(r)^{-1} \lambda(r t), e\right) \in T,
$$

and we may continue working with this element. Now recall that we have picked two distinct elements $c, d \in G_{e} \backslash\{e\}$ such that $\lambda(c)=\gamma_{1}$ and $\lambda(d)=\gamma_{2}$, the generators of $H$. If $m \geq 1$ is the order of $h_{0}$ (recall that $H$ is periodic), the elements

$$
\begin{aligned}
& \left(e, h_{0}, e\right)^{m} c\left(e, h_{0}, e\right)^{m}=\left(e, 1_{H}, e\right)(c, \lambda(c), e)=\left(e, p_{e, c} \lambda(c), e\right)=\left(e, \gamma_{1}, e\right) \\
& \left(e, h_{0}, e\right)^{m} d\left(e, h_{0}, e\right)^{m}=\left(e, 1_{H}, e\right)(d, \lambda(d), e)=\left(e, p_{e, d} \lambda(d), e\right)=\left(e, \gamma_{2}, e\right)
\end{aligned}
$$

both belong to $T$. Since $p_{e, e}=1_{H}$, the mapping $H \rightarrow H_{e}=\{e\} \times H \times\{e\}$ defined by $h \mapsto(e, h, e)$ is an isomorphism; therefore, $\left(e, \gamma_{1}, e\right),\left(e, \gamma_{2}, e\right) \in T$ clearly implies $H_{e} \subseteq T$. Furthermore, for each $\ell \in L$ and $r \in R$ we have $\ell(\{e\} \times H \times\{e\}) r \subseteq T$. The left-hand side of this inclusion is just $\{\ell\} \times H \times\{r\}$, because for any $h \in H$ we have

$$
\ell(e, h, e) r=(\ell, \lambda(\ell) h \lambda(r), r),
$$

and the function $x \mapsto \lambda(\ell) x \lambda(r)$ is a bijection of $H$ onto itself. So, $L \times H \times R \subseteq$ $T$ and thus $T=\Sigma_{S}$, as required.

\section{Proof of Theorem A}

As we have already remarked, Theorem B implies the backward implication in Theorem A: any finite group with at least three elements is big in the class of all semigroups.

To complete the proof of Theorem A we need to demonstrate that the trivial group and the cyclic group of order 2 are not big. First we deal with the trivial group.

Proposition 3. Let $S$ be an infinite semigroup containing an idempotent $e$. Then $S$ has a proper subsemigroup $S^{\prime}$ with at least two elements such that $e \in S^{\prime}$.

Proof. Suppose to the the contrary that $S$ is a witness for $\{e\}$. Both $e S$ and $S e$ are subsemigroups containing $e$, so that $e S, S e \in\{S,\{e\}\}$. If $e S=S e=$ $S$ (respectively, $e S=S e=\{e\}$ ) the idempotent $e$ is in fact the identity (respectively, zero) of $S$. In either of these cases, for any $s \in S \backslash\{e\}$ we have $S=\langle e, s\rangle=\left\{e, s, s^{2}, \ldots\right\}$, and $\left\{e, s^{2}, s^{4}, \ldots\right\}$ is a proper subsemigroup, a contradiction. If $e S=\{e\}$ and $S e=S$ (respectively, $e S=S$ and $S e=\{e\}$ ) 
then $S$ is a left (respectively, right) zero semigroup, and every subset is a subsemigroup, a contradiction again.

Before turning our attention to the cyclic group of order 2, we prove yet another result of a general character, which refines the information supplied by Lemma 1, and which might be quite useful in further investigations of big semigroups.

Lemma 4. Let $S$ be a big semigroup, and let $T$ be any witness for $S$. Let $J$ be the unique $\mathscr{J}$-class of $T$ containing $T \backslash S$. Then $J$ contains a $J$-primitive idempotent, that is, a minimal element in the restriction of the Rees order of idempotents of $T$ to $J \cap E(T)$.

Proof. We break the proof up into the following steps:

(i) There exist $a, b \in J$ such that $a b \in J$.

(ii) There exists $t \in J$ such that $t^{n} \in J$ for all $n \in \mathbb{N}$.

(iii) $J$ contains an idempotent.

(iv) $J$ contains a $J$-primitive idempotent.

(i) Let $u \in J \backslash S$ be arbitrary. Since $T$ is a witness for $S$, we must have

$$
\langle S \cup\{u\}\rangle=\bigcup_{n=0}^{\infty} S^{1}\left(u S^{1}\right)^{n}=T .
$$

From finiteness of $S$ it follows that $S^{1} u S^{1}$ is finite, and, since $J$ is infinite, we have

$$
S^{1} u S^{1} u S^{1} \cap J \neq \emptyset .
$$

If $s_{1} u s_{2} u s_{3}$ is any element of this set, and if we let $a=s_{1} u, b=s_{2} u s_{3}$, we clearly have $a, b, a b \in J$, as required.

(ii) Let $t_{1}, t_{2} \in T^{1}$ be such that $a=t_{1} a b t_{2}$. Then $a=t_{1}^{n} a\left(b t_{2}\right)^{n}$ for all $n \in \mathbb{N}$, and it is easy to verify that $t=b t_{2}$ satisfies the requisite property.

(iii) If $t \in S$ then, since $S$ is finite, $t$ has an idempotent power, and we are done. Otherwise $\langle S \cup\{t\}\rangle=T$. From $t, t^{2} \in J$ we have $t=x t^{2} y$ for some $x, y \in T^{1}$. Writing $x$ and $y$ as alternating products of elements of $S$ and $t$, we see that $t$ can be written as $t=s_{1} t u_{1} t s_{2}$ for some $s_{1}, s_{2} \in S^{1}$ and $u_{1} \in T^{1}$. Substituting repeatedly this expression into itself we obtain

$$
t=s_{1}^{n} t u_{n} t s_{2}^{n},
$$

for all $n \in \mathbb{N}$ and some $u_{n} \in T^{1}$. Since $S$ is finite there exists $n \in \mathbb{N}$ such that both $s_{1}^{n}$ and $s_{2}^{n}$ are idempotents. But then (5.1) yields $s_{1}^{n} t=t s_{2}^{n}=t$, and hence $t=t u_{n} t$. It follows that $t u_{n}$ is an idempotent and $t u_{n} \in J$, as required.

(iv) Aiming for contradiction, suppose that $J$ does not contain a $J$ primitive idempotent. Then $J$ must contain an infinite descending chain of idempotents. Since $S$ is finite there must exist two idempotents $f, f^{\prime} \in J \backslash S$ with $f<f^{\prime}$, i.e. $f f^{\prime}=f^{\prime} f=f$. From $T=\langle S \cup\{f\}\rangle$ it follows that $f^{\prime}=s f u$, with $s \in S^{1}, u \in T^{1}$. But then

$$
f^{\prime}=s f u=s f^{\prime} f u=s^{2} f u f u=s^{2} f^{\prime}(f u)^{2}=\cdots=s^{n} f^{\prime}(f u)^{n}=\ldots .
$$


Choosing $s^{n}$ to be idempotent, we have $f^{\prime}=s^{n} f^{\prime}$, which in turn implies

$$
f^{\prime} s^{n} f^{\prime}(f u)^{n}=f^{\prime}(f u)^{n}=(f u)^{n},
$$

and hence

$$
f=f f^{\prime}=f(f u)^{n}=(f u)^{n}=f^{\prime}
$$

a contradiction.

Proposition 5. The cyclic group of order 2 is not a big semigroup.

Proof. Assume to the contrary, so that $C_{2}=\{e, a\}$ (with $a^{2}=e$ ) has a witness $T$. By Lemma $1, T \backslash C_{2}$ is contained in a single $\mathscr{J}$-class $J$ of $T$, so we have two possibilities: either $T=J$ is simple, or $T$ has precisely two $\mathscr{J}$-classes, namely $C_{2}$ and $J$. In either case $J$ is the kernel of $T$, and, by Lemma 4 , contains a $J$-primitive idempotent, which, in fact, must also be $T$-primitive, implying that $J$ is completely simple.

Consider first the case when $T=J$. Then, by the Rees-Suškevič Theorem, $T \cong \mathcal{M}(I, G, \Lambda, P)$ for some group $G$, index sets $I, \Lambda$ and a matrix $P$ over $G$. So, any subgroup of $T$ (and thus any instance of $C_{2}$ ) is contained in one of the maximal subgroups $G_{i, \mu}=\{i\} \times H \times\{\mu\}$ for some $i \in I, \mu \in \Lambda$, all of them being isomorphic to $G$. Now if $G$ is an infinite group, then for any its cyclic subgroup of order 2 there is a proper subgroup $G_{1}$ properly containing that cyclic subgroup. This shows that no subgroup of $G_{i, \mu}$ of order 2 can be a maximal subsemigroup of $G_{i, \mu}$, let alone of $T$. So, $G$ must be a finite group, whence at least one of the sets $I, \Lambda$ are infinite. However, note that for each element $\left(j, h^{\prime}, \nu\right)$ of $T$, any element of the subsemigroup of $S$ generated by $H_{i, \mu} \cup\left\{\left(j, h^{\prime}, \nu\right)\right\}$ must belong to $H_{i, \mu} \cup H_{j, \mu} \cup H_{i, \nu} \cup H_{j, \nu}$, rendering $\left\langle H_{i, \mu} \cup\left\{\left(j, h^{\prime}, \nu\right)\right\}\right\rangle=T$ impossible. A contradiction.

Therefore, $T$ must have precisely two $\mathscr{J}$-classes: $C_{2}$ and $J$, the latter being a completely simple ideal of $T$. In particular, $T$ has an idempotent $f \neq e$, whence we have $T=\langle a, f\rangle$, and $e$ can be assumed to be the identity element of $T$, because otherwise $e T e$ would be a proper subsemigroup of $S$ that properly contains $C_{2}$ (as $e J e$ is a non-empty subset of $J$ ). Hence, each element of $J$ can be expressed as an alternating product of $a$ and $f$. On the other hand, faf $\in J$, so $f a f \mathscr{J} f$, implying $f=t_{1}(f a f) t_{2}=f t_{1} f a f t_{2} f$ for some $t_{1}, t_{2} \in J^{1}$. The factors $t_{1}, t_{2}$ are either empty, or expressible as alternating products of $a$ and $f$, thus we have

$$
(f a f)^{k}=\underbrace{f a f \cdots f a f}_{k+1 \text { times } f}=f
$$

for some $k \geq 1$. Hence any alternating product of $a$ and $f$ of length at least $2 k+2$ can be reduced to a shorter product; in other words, $J$ is exhausted by such alternating products of length at most $2 k+1$. It follows that $J$ is finite, as well as $T$ - a contradiction.

This completes the proof of Theorem A. 


\section{Concluding remarks}

The finite semigroups under the scope of Theorem B are not the only big semigroups. Indeed, for two semigroups $P$ and $Q$ define their ordinal sum $P[Q]$ on $P \cup Q$ to be the semigroup obtained by retaining the multiplication operations from $P$ and $Q$ and setting $p q=q p=q$ for all $p \in P, q \in Q$. Now if $P$ is a big semigroup and $Q$ is any finite semigroup, then $P[Q]$ is big as well, because if $T$ is a witness for $P$, then $T[Q]$ is a witness for $P[Q]$.

Corollary 6. Let $S$ and $Q$ be arbitrary finite semigroups such that the kernel of $S$ has a subgroup with at least three elements. Then the semigroup $S[Q]$ is big.

The following goal seems to be distant at the moment, but we formulate it nevertheless.

Problem. Characterise all big semigroups.

\section{Acknowledgment}

We are grateful to our colleague Petar Marković for drawing the first author's attention to the paper [3] and suggesting the study of big semigroups as a potential research topic.

\section{References}

[1] S. I. Adyan and I. G. Lysionok, On groups all of whose proper subgroups are finite cyclic. Izv. Akad. Nauk SSSR, Ser. Mat. 55 (1991), 933-990 (in Russian; English transl. Math. USSR-Izv. 39 (1992), 905-957).

[2] A. H. Clifford and G. B. Preston, Algebraic Theory of Semigroups, Vol. I. American Mathematical Society, Providence, 1961.

[3] R. Freese, J. Ježek and J. B. Nation, Lattices with large minimal extensions. Algebra Universalis 45 (2001), 221-309.

[4] N. Graham, R. Graham and J. Rhodes, Maximal subsemigroups of finite semigroups. J. Comb. Theory 4 (1968), 203-209.

[5] J. M. Howie, Fundamentals of Semigroup Theory. Oxford University Press, New York, 1995.

[6] A. Yu. Ol'shanskiǔ, Groups of bonded period with subgroups of prime order. Algebra i Logika 21 (1982), 553-618 (in Russian; English transl. Algebra and Logic 21 (1982), 369-418).

[7] M. Petrich, The translational hull of a completely 0-simple semigroup. Glasgow Math. J. 9 (1968), 1-11.

I. Dolinka

Department of Mathematics and Informatics

University of Novi Sad

Trg Dositeja Obradovića 4

21101 Novi Sad, Serbia

e-mail: dockie@dmi.uns.ac.rs 
N. Ruškuc

School of Mathematics and Statistics

University of St Andrews

St Andrews KY16 9SS, Scotland, UK

e-mail: nik@mcs.st-and.ac.uk 